\title{
Erratum to: Aspirin and non-aspirin NSAIDs increase risk of colonic diverticular bleeding: a systematic review and meta-analysis
}

\author{
Hiroki Yuhara · Douglas A. Corley · Fumio Nakahara • \\ Takayuki Nakajima · Jun Koike • Muneki Igarashi • \\ Takayoshi Suzuki • Tetsuya Mine
}

Published online: 9 August 2014

(C) Springer Japan 2014

Erratum to: J Gastroenterol (2014) 49:992-1000

DOI 10.1007/s00535-013-0905-z

The name of the seventh author should be given as Takayoshi Suzuki, not Takayoshi Suauki.

The online version of the original article can be found under doi:10.1007/s00535-013-0905-z.

H. Yuhara $(\bowtie) \cdot$ F. Nakahara $\cdot$ T. Nakajima $\cdot$ J. Koike .

M. Igarashi - T. Suzuki - T. Mine

Division of Gastroenterology and Hepatology, Department of Internal Medicine, Tokai University School of Medicine,

143 Shimokasuya, Isehara 259-1193, Japan

e-mail: hirokiyuhara@gmail.com

T. Mine

e-mail: tetsu-m@is.icc.u-tokai.ac.jp

D. A. Corley

Division of Research, Kaiser Permanente, Oakland, USA 